\title{
ZU DEN REDUPLICIERTEN PRAETERITA.
}

1. Zarncke, Beitr. 15, 350 und Holz (Urgermanisches geschlossenes $\bar{e}$, Leipzig, 1890) haben eine anzahl von belegen für das ahd. $r$-präteritum ubersehen, aus denen hervorgeht, dass dieser typus keineswegs auf Reichenau beschränkt war, wie Zarncke meinte. In den verschiedenen handschriften der Prudentiusglossen begegnet zunächst an zwei stellen das präteritum stiriz zu stôzan. Gl. 2, 542, 7 lesen wir in einer Pressburger handschrift prosubigit stiriz, wobei das deutsche wort am rande mit $i .=i d$ est widerholt ist; die glosse kehrt wider in zwei handschriften der nummer DCCXC bei Steinmeyer-Sievers, einer Pariser und einer Munchener aus St. Emmeram (letztere von Graff als Prud. 1 citiert) in der gestalt stirz, einer form, die der Regensburger copist zuerst nicht verstanden und durch spurnta ersetzt hatte, aber dann doch noch am rande nachtrug, Gl. 2, 444, 22. Dagegen stellen andere schreiber, und ihnen schliesst sich an der eben citierten stelle auch der herausgeber Steinmeyer an, die regelmässige form stiez her: 508, 1. 536, 34. Die andere glosse, nicht weit von der ersten entfernt, lautet in dem erwähnten codex Apponyianus (Pressburg) Gl. 2, 542, 19 pupugerat stiriz, wofur die ubrigen schreiber die gewöhnliche form einsetzen, 508, 25. 536, 43. Dazu kommt ferner die form steraz, die der Tegernseer schreiber der Vergilglossen des Munchener codex 18059 als glosse zu arietat Aen. 11, 890 in seiner vorlage vorfand, aber nachträglich in stiaz corrigierte, Gl. 2,669,50. In der gleichen handschrift lesen wir 670, 16 proterret farsterc, von anderer hand als die vorige glosse, aber gewiss aus der gleichen varlage, deren farsterz oder farsteraz dem copisten unverständlich war. Die formen steraz (= älterem sterôz) und stiriz sind damit für das bai- 
rische sprachgebiet sicher gestellt. Zweifelhafter ist ein $r$-präteritum von lâzan in einer Trierer handschrift Gl. 2, 33, 1 indulsere anagelierzon, weil das ie es in der tat nahelegt, das $r$ nur als schreibfehler für $z$ zu betrachten. Was die zeit anlangt, so sind die genannten handschriften alle jung, aus dem ende des 10. und dem anfange des 11. jahrhunderts, und auch die originalglossierungen auf denen sie beruhen, können nicht sehr weit zurïckverlegt werden. Denn Prudentius und Virgil sind erst ziemlich spät in den bereich des schulmässigen studiums gezogen worden. Man muss den kritischen apparat zu der glossenausgabe abwarten, ehe man auf diese fragen genauer eingehen kann. Zur erklärung der form stiriz bemerke ich nur noch, dass sich das $i$ der reduplicationssilbe mit leichtigkeit aus dem tiefstufigen plural *stiruzzum herleiten lässt, da durch das folgende $u$ der lubergang von $e$ in $i$ gefordert wird, wie in Behaghel-Neumanns Literaturbl. 1887, s. 108 dargelegt ist. Zur ergänzung meiner daselbst gegebenen ausfuhrungen fuge ich bei, dass auch die ausnahmen, von denen Braune, Ahd. gr. ${ }^{2} 19$ einige aufführt, unter einem gesetze stehen. Der libergang von $e$ in $i$ vor $u$ unterbleibt nämlich uberall da, wo auf das $u$ ein brechung bewirkender rocal $(a, \hat{o}, \hat{e})$ folgt oder folgte. Daher

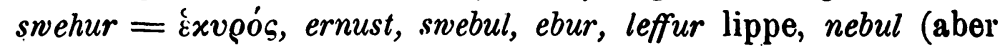
Nipulunc nibulen), nebst nemunga und den ubrigen worten gleicher bildung. Zarncke a. a. o. vermisst einen beleg für das part. giscriran zu scrian; er steht Gl. 2, 775,6 conclamata erscrirena. Die glosse recrementum spirin 2, 532, 50 gehört kaum zu spian, sondern meint wol spriu, nach massgabe der ubrigen handschriften.

2. Dass das prät. otarseu Hel. $2545 \mathrm{C}$ auf angelsächsischem einflusse beruhe, ist eine so baltlose annahme von Holz, dass Sievers Beitr. 16, 255 mit recht daruber zur tagesordnung tibergeht. Wenn Holz der mittelniederländischen grammatik etwas mehr aufmerksamkeit geschenkt hätte, als es der fall gewesen zu sein scheint (die wichtigen mnl. formen der reduplicierten präterita finden bei ihm keinerlei berlicksichtigung), so würde er gefunden haben, dass das seu des Werdener schreibers oder dichters der grenzlage seines klosters gemäss seine genaue entsprechung hat in mnl. sieu, der in dieser sprache einzig vorkommenden form, die mit crieu, wieu, grieu zu craien, waien, 
groeien auf gleicher linie steht, Franck § 154. Desgleichen deckt sich heu mit mnl. hieu, pl. hieuwen. - Mangelhafte kenntnis des tatsäcblichen tritt auch auf s. 36 hervor, wo über erian gesprochen wird. Denn die präteritalform uor, die Holz vermisst, ist ja tatsächlich vorhanden, wie aus Graff 1, $403 \mathrm{zu}$ lernen war, sofern man sich nur die muhe genommen hätte, die daselbst citierten stellen nachzuschlagen. Denn Gl. 1, 386, $9 \mathrm{ff}$. schwanken die handschriften in einer glosse zu arassetis zwischen ir-ierit, der regelmässigen form, und ir-uorit, ir-uoret, woraus wider andere das sinnlose iruuorit (das nur zu varan gehören könnte) machen. Man hat also das verhältnis swerien : swuor wirklich nachgebildet und dieser umstand beweist eben, dass die von Holz angenommene präsensform mit länge ( $\mathrm{futr}$ die er sich auf Bremer beruft, der aber nicht Beitr. 11, 107, sondern s. 283 daruber handelt) ein blosses phantasiegebilde ist. Das präsens lautet in keiner quelle, sei sie alt oder jung, je anders als erren, erien (d. i. erijen). Ob diese form in das system, das sich der oder jener ausgedacht hat, passt oder nicht, ist gleichgultig. Die tatsachen allein sind massgebend und sie zu erklären ist die aufgabe der sprachwissenschaft, nicht sie zu ignorieren oder durch allerlei kunststuckchen bei seite $\mathrm{zu}$ schieben. BASEL, august 1891.

RUDOLF KÖGEL.

\section{IDIS UND WALKÜRE.}

Das wort itis, alts. idis, ags. ides hat man zu der wurzel gestellt, die in eit, ital u. s. w. zur erscheinung kommt und hat also darin den begriff des glänzenden, leuchtend schönen ausgedrickt gefunden. Diese etymologie scheint in anbetracht der seltsamen formen, in denen das wort auftritt, nicht haltbar zu sein. Denn ausser der ersten silbe, wo es nicht weiter suffällig wäre, erscheint auch die zweite in doppelter quantität. Dass die erste silbe des alts. idis, ags. ides in der regel kurz ist, ergibt sich aus den gesetzen des stabreimenden verses; auf hochdeutschem boden scheint aber auch die länge gegolten $\mathrm{zu}$ 